\title{
Polymer Chain Dynamics I. Estimation of Orientational Auto-Correlation Function for Polymer Chains
}

\author{
Fumiaki TsUNOMORI and Hideharu UsHIKI \\ Graduate School of Bio-Applications and Systems Engineering, Tokyo University of \\ Agriculture and Technology, Fuchu, Tokyo, 183 Japan
}

(Received October 23, 1995)

\begin{abstract}
Fluorescence anisotropy decay curves of polystyrene labeled with an anthryl group in the middle of a main chain (PSAPS) were measured by the time-resolved fluorescence depolarization method. We studied an adequate orientational auto-correlation functions (OACF) to explain fluorescence anisotropy decay curves of PSAPS. The goodness of fitting to measured anisotropy decays is evaluated by an original fitting program based on Wahl's argolithm. However it was difficult to distinguish the most adequate OACF from many reported ones only by the comparison of the values of the residual sum of squares. Consideration of the meaning of variable parameters contained in many OACF reported has been done from the viewpoint that OACF should refrect the distribution of relaxation rate. Furthermore, a novel method for estimation of $\mathrm{OACF}$ called $\chi^{2}$-map method was used. As a result the hyperbolic function is one of the most adequate OACF in order to express effectively the fluorescence anisotropy decay curves of PSAPS chains.

KEY WORDS Fluorescence Depolarization / Polystyrene / $\chi^{2}$-Map / Long Time Tail / Hyperbolic

Function /
\end{abstract}

It is important to make a realistic description of the orientational relaxation of a polymer chain in order to understand dynamic properties of polymers because the orientational relaxation of polymer segments is the most elemental process for conformational change among the vast number of relaxation modes of polymer back bone. During the last twenty years, theoretical and experimental studies of orientational relaxation of a polymer chain in dilute solution have been reported by many authors. ${ }^{1-7}$ In spite of many efforts, a satisfactory description has not been proposed, because most studies approximated the orientational relaxation to consist of a few relaxation modes.

Various orientational auto-correlations of polymer main chain were measured by admirable techniques of experiments such as dielectric relaxation, ${ }^{8} \mathrm{NMR},{ }^{9} \mathrm{ESR},{ }^{10}$ and fluorescence depolarization method. ${ }^{1,6,11}$ Especially, the time-resolved fluorescence depolarization method is very powerful to estimate various molecular motions of a polymer chain because a fluorescence anisotropy decay curve (FADC) of a chromophore incorporated into a polymer chain refers to orientational relaxation motion of a polymer chain. ${ }^{7,12,13}$

Valeur et $a .^{1,14,15}$ were the first to explain FADC measured by the time-resolved fluorescence depolarization method on the basis of a theoretical model which describes the orientational relaxation of polystyrene main chain obeying 3-bond motion. With their reports as a trigger, many researchers could get clues for the study of the orientational relaxation motion of a polymer main chain. Viovy et al. discussed an appropriate orientational auto-correlation function (OACF) to interpret the local chain motion, and proposed the generalized diffusion and loss model, ${ }^{5}$ which is derived by extension of Hall and Helfand's model, ${ }^{4}$ as the best $\mathrm{OACF}$. The main point at issue on the local motion started by Valeur et al. was essentially to make a realistic description of the orientational relaxation motion of a main chain of an isolated polymer. However progress about this problem could be hardly made from Viovy's work.

In recent years, characteristics of bulk polymer and polymer chain dissolved in high viscosity solvent were estimated on the grounds of properties of an isolated polymer chain. Waldow et al. pointed out that it is diffcult to record the complete form of FADC under high viscosity system. ${ }^{11}$ They further noted that unrealistic interpretations about the local motion of bulk polymer are obtained by the GDL model analysis. Therefore, Waldow et al. discussed the local motion of a polymer chain associated with the apparent activation energy which is estimated by the temperature dependence of the averaged relaxation time $\tau_{\mathrm{c}}$

$$
\tau_{\mathrm{c}}=\int_{0}^{\infty} r(t) \mathrm{d} t
$$

where $r(t)$ is a measured FADC. The main aim of the local motion analysis by the mean relaxation time $\tau_{\mathrm{c}}$, that is, 'model free approach' is to discuss the mean properties of a polymer chain without taking into account details of local motion.

In this work, we discuss an adequate OACF in order to make a realistic description of the orientational relaxation motion of polymer main chain.

\section{EXPERIMENTAL}

\section{Sample}

Polystyrene with an anthryl group in the middle of its chain (PSAPS) used in this study was synthesized in the same manner as reported previously. ${ }^{16}$ Styrene was polymerized anionically in tetrahydrofuran with cumyl potassium as the initiator under a high vacuum system. PSAPS chains were given by the direct attack of living polystyryl monoanions with stoichiometric amount of 9,10-bis(chloromethyl)anthracene. Chemical structure of PSAPS is shown in Figure 1. The values of the number-average molecular weight $M_{n}$ and molecu- 


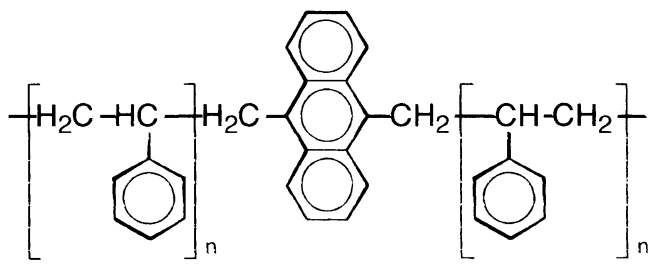

Figure 1. Chemical structure of PSAPS

lar-weight distribution $M_{w} / M_{n}$ measured with a gel permeation chromatography are $3.3 \times 10^{5}$ and 1.39 , respectively. Percentage of the anthryl group in the polymers relative to theoretical content measured with an ultraviolet spectrophotometer is $95 \%$.

PSAPS was dissolved in a mixed solvent of tripropionin (glyceryl tripropionate) and ethyl acetate. The volume fraction of tripropionin was 0.94 , and the concentration of polymer was kept less than $0.1 \mathrm{wt} \%$ at the final step of preparation. Sample solutions degassed by several freeze-pump-thaw cycles were enclosed in Pyrex tube cells.

\section{Measurement}

The absorption, excitation, emission, and depolarization spectra, and time-resolved emission decay curves of PSAPS were measured by the PLASMA system. ${ }^{17}$ The PLASMA system is a novel measurement system which consists of various spectroscopic apparatus together with a personal computer network supported by many original electric circuits and programs. Excitation and emission spectra were corrected by a concentrated solution of rhodamine $\mathrm{B}$ to compensate for the spectroscopic sensitivity of optical equipment. The excitation and emission wavelengths were set at 384 and $430 \mathrm{~nm}$, respectively.

Fluorescence decay curves $F_{\text {obs }}(t)$ and FADC's $r_{\text {obs }}(t)$ were calculated by

$$
\begin{aligned}
& F_{\mathrm{obs}}(t)=I_{\mathrm{VV}}(t)+2 G I_{\mathrm{VH}}(t) \\
& r_{\mathrm{obs}}(t)=\frac{I_{\mathrm{VV}}(t)-G I_{\mathrm{VH}}(t)}{I_{\mathrm{VV}}(t)+2 G I_{\mathrm{VH}}(t)}
\end{aligned}
$$

where $G$ is the compensating factor for anisotropic sensitivity of the photomultiplier of the time-resolved fluorophotometer (Horiba: NAES-1100). $G$ is defined by

$$
G=\int I_{\mathrm{HV}}(t) \mathrm{d} t / \int I_{\mathrm{HH}}(t) \mathrm{d} t
$$

$I_{\mathrm{VV}}(t), I_{\mathrm{VH}}(t), I_{\mathrm{HH}}(t)$, and $I_{\mathrm{HV}}(t)$ indicate decay curves of polarized fluorescence intensity measured through a band path filter (Toshiba: KL43) selected according to absorption, excitation, and emission spectra recorded by the fluorescence spectrophotometer (Hitachi: 650-60). Subscripts $\mathrm{V}$ and $\mathrm{H}$ represent parallel and perpendicular directions to the vertical line. The first suffix indicates the direction of incident light and the second, to emitted light.

\section{Calculation}

In general, $F_{\text {cal }}(t)$ and $r_{\text {cal }}(t)$ are reproduced by

$$
\begin{gathered}
F_{\text {cal }}(t) \sim \int_{0}^{t} P(T) S(t-T) \mathrm{d} T \\
r_{\mathrm{cal}}(t) \sim \frac{\int_{0}^{t} P(T) D(t-T) S(t-T) \mathrm{d} T}{\int_{0}^{t} P(T) S(t-T) \mathrm{d} T}
\end{gathered}
$$

where $P(t), S(t)$, and $D(t)$ represent a function of the temporal characteristics of the apparatus, a fluorescence decay curve corresponding to an infinitely short excitation and OACF, respectively. $S(t)$ is a whole fluorescence decay curve which is usually the sum of exponential functions, written as,

$$
S(t)=\sum_{i=1}^{m} a_{i} \exp \left(-t / \tau_{i}\right)
$$

where $\tau_{i}$ s are fluorescence decay constants. We assumed that $P(t)$ and $S(t)$ are an exciting light pulse and double exponential function, respectively. For the case of a double exponential function, the average fluorescence lifetime $\bar{\tau}_{f}$ is obtained by

$$
\bar{\tau}_{f}=\frac{a_{1}}{a_{1}+a_{2}} \tau_{1}+\frac{a_{2}}{a_{1}+a_{2}} \tau_{2}
$$

We made a deconvolution and curve fitting programs by use of Pascal language (Borland: Turbo PASCAL). Deconvolution and curve fitting procedures are based on Wahl's method ${ }^{18}$ and the quasi-Marquardt algorithm, ${ }^{19}$ respectively. These calculations were carried out on a personal computer (NEC: PC-9801RA) with a co-processor for numerical calculations (Intel: 80387).

The fitting was evaluated by a value of the residual sum of squares, $\chi^{2}$. The program computes adequate values of variable parameters of a trial function until the variation of $\chi^{2}$ become less than $1 \times 10^{-6}$. On determing the value of parameters $a_{i}$ and $\tau_{i}$ in $S(t), \chi^{2}$ was calculated by,

$$
\chi^{2}=\frac{1}{t_{2}-t_{1}-4} \int_{t_{1}}^{t_{2}} w(t)\left[F_{\text {obs }}(t)-F_{\text {cal }}(t)\right]^{2} \mathrm{~d} t
$$

where $w(t), t_{1}$, and $t_{2}$ indicate a weighting function at time $t$, initial and final time of the fitting range, respectively. The weighting function adopted for $F_{\text {cal }}(t)^{18}$ is,

$$
w(t)=\frac{1}{I_{\mathrm{VV}}(t)+4 G^{2} I_{\mathrm{VH}}(t)}
$$

In contrast, on estimating the value of parameters in $D(t)$, eq 9 was substituted by

$$
\chi^{2}=\frac{1}{t_{2}-t_{1}-m} \int_{t_{1}}^{t_{2}} w(t)\left[r_{\mathrm{obs}}(t)-r_{\mathrm{cal}}(t)\right]^{2} \mathrm{~d} t
$$

where $m$ is the number of variable parameters of the function $D(t)$. The weighting function was replaced by ${ }^{18}$

$$
w(t)=\frac{3 F_{\mathrm{obs}}(t)}{1+G+3 G r_{\mathrm{obs}}(t)-3 r_{\mathrm{obs}}(t)^{2}-2(2 G-1) r_{\mathrm{obs}}(t)^{3}}
$$




\section{RESULTS AND DISCUSSION}

\section{Decay Curves and Features of $O A C F$}

Average fluorescence lifetimes $\bar{\tau}_{f}$ are shown in Table I. $\bar{\tau}_{f}$ of an anthryl group incorporated in the center of polymer back bone seemed constant in the range of temperature from $20^{\circ} \mathrm{C}$ to $42.5^{\circ} \mathrm{C}$, though fluorescence lifetime generally decreased with increasing temperature. This suggests that we can treat fluorescence lifetime as constant in this study because of narrow temperature range. The value of $\bar{\tau}_{f}$ is about $9 \mathrm{~ns}$.

FADC measured at $30^{\circ} \mathrm{C}$ and calculated anisotropy curve by use of the hyperbolic function are shown in Figure 2. The fluorescence anisotropy curve decayed sharply at the initial stage of relaxation, and had a long time tail at the final stage. In general, small molecules such as 9,10-diphenylanthracene rotate isotropically, so that fluorescence anisotropy of the molecules obeys the exponential law,

$$
r(t) \sim \exp \left(-\frac{t}{\tau}\right)
$$

because small molecule is regarded as a rigid and spherical rotator, where $t$ is time, and $\tau$ is an effective rotational correlation time. A probe molecule incorporated in the back bone of the polymer chain cannot rotate similarly to small molecules but wobble in the wake of orien-

Table I. Fluorescence lifetime calculated by a double exponential decay function

Values of fluorescence lifetime are not varied by temperature. Average value of lifetime is $9.2 \mathrm{~ns}$

\begin{tabular}{lcccccc}
\hline Temp $/{ }^{\circ} \mathrm{C}$ & 20.0 & 25.0 & 30.0 & 37.5 & 40.0 & 42.5 \\
\hline Lifetime/ns & 9.1 & 9.0 & 9.0 & 9.3 & 9.2 & 9.3 \\
\hline
\end{tabular}
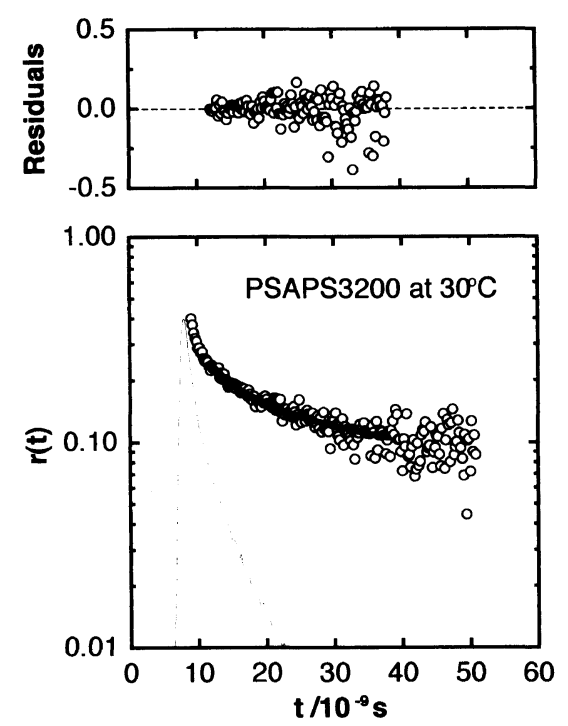

Figure 2. Semi-log plots of a FADC of an anthryl group incorporated in PSAPS3200 chain measured at $30^{\circ} \mathrm{C}$. Thick line is a calculated curve by use of the hyperbolic function, thin line is a response function of the apparatus, and circles are measured anisotropy decay curves. Calculated curve is obtained by convolution fitting of the hyperbolic function with the response function. Fitting calculation was carried out successfully in the fitting range because residuals are symmetrically distributed around the zero line. tational relaxation motion of a polymer back bone. Thus fluorescence anisotropy of a probe molecule attached to the center of a polymer chain reflects a great number of relaxation modes. In order to understand the dynamics of conformational change of polymer chain on the basis of fluorescent anisotropy measurement, it is important to determine a trial function taking into account a vast number of local modes of the chain.

OACFs to describe FADCs are listed in Table II. The 'Single' function is only useful in an ideal system which is not needed to consider the dispersion of relaxation rate. The 'Double' function consists of two equivalent terms of exponential function: one corresponds to a fast relaxation mode and another to a slow one. Jones et al. ${ }^{2}$ proposed 'JS' function comprised of many exponential terms. Appropriate approximation gives a double exponential function whose prefactors are fixed.

'VJGM' function is derived by Valeur et al. ${ }^{14}$ They discussed local motion of polymer chain based on the 3-bond motion on a tetrahedral lattice. They concluded that, at long time, anisotropy decay curve obeys, ${ }^{15}$

$$
r(t) \sim\left(\frac{t}{\tau}\right)^{-1 / 2}
$$

Here it should be emphasized that VJGM function allows us to predict behavior dependent on the power law.

Hall et al. ${ }^{4}$ reported a useful function ('HH' function) based on two-state model. Extending ' $\mathrm{HH}$ ' function, 'GDL' (Generalized Diffusion and Loss) function is derived by Viovy et al. ${ }^{5} \mathrm{HH}$ and GDL functions regard anisotropy relaxation of a probe molecule in the polymer chain as the product of local and global modes. The former is the product of an exponential and modified Bessel functions, the latter is an exponential function.

Weber et $a l .{ }^{20}$ presented a function ('WH' function) consisting of a constant term and decay one which is the same as $\mathrm{HH}$ function. For molecular motion in membranes, Kinoshita et al. ${ }^{21}$ derived 'WC' (Wobblingin-Cone) function having a constant and single exponential term. The feature of these functions is that global modes of the chain is treated as a constant parameter.

'BY' function derived by Bendler et al. ${ }^{3}$ considers dispersion of relaxation frequency. Two relaxation times in its function refer to fast and slow cutoff frequencies. However it is difficult to relate these cutoff times to molecular times.

Table II. Orientational auto-correlation functions in this study

\begin{tabular}{ll}
\hline Model & Function \\
\hline Single & $\exp (-t / \tau)$ \\
Double & $a_{1} \exp \left(-t / \tau_{1}\right)+a_{2} \exp \left(-t / \tau_{2}\right)$ \\
JS & $0.578 \exp \left(-t / \tau_{1}\right)+0.422 \exp \left(-t / \tau_{2}\right)$ \\
VJGM & $\exp \left(-t / \tau_{1}\right) \exp \left(t / \tau_{2}\right) \operatorname{erfc}\left(\sqrt{t / \tau_{2}}\right)$ \\
HH & $\exp \left(-t / \tau_{1}\right) \exp \left(-t / \tau_{2}\right) I_{0}\left(t / \tau_{2}\right)$ \\
GDL & $\exp \left(-t / \tau_{1}\right) \exp \left(-t / \tau_{2}\right)\left(I_{0}\left(t / \tau_{2}\right)+I_{1}\left(t / \tau_{2}\right)\right)$ \\
WH & $(1-a) \exp \left(-t / \tau_{1}\right) \exp \left(-t / \tau_{2}\right) I_{0}\left(t / \tau_{2}\right)+a$ \\
WC & $(1-a) \exp (-t / \tau)+a$ \\
BY & $1 / 2 \sqrt{\pi}\left(\operatorname{erfc}\left(\sqrt{t / \tau_{1}}\right)-\operatorname{erfc}\left(\left(\sqrt{t / \tau_{2}}\right)\right) /\left(\sqrt{t / \tau_{2}}-\sqrt{t / \tau_{1}}\right)\right.$ \\
KWW & $\exp \left(-(t / \tau)^{\beta}\right)$ \\
Hyperbolic & $(t / \tau)^{-\beta}$ \\
Hyperbolic $\cdot \mathrm{KWW}$ & $(t / \tau)^{-\beta} \exp \left(-(t / \tau)^{\beta}\right)$ \\
\hline
\end{tabular}


It is well known that hyperbolic and $\mathrm{KWW}^{22,23}$ functions are able to express long time tail of relaxation phenomena. ${ }^{24,25}$ Relaxation phenomena of amorphous materials obeying $\mathrm{KWW}$ and/or the hyperbolic functions are discussed from a viewpoint of the fractal geometry or the fractal time stochastic process. ${ }^{26} \mathrm{KWW}$ and the hyperbolic function are employed to interpret the asymmetric and symmetric spectrum of a complex dielectric constant as a function of frequency in amorphous materials. ${ }^{27,28}$ In particular, the hyperbolic function is useful to analyze the frequency dependence of complex dielectric constant in polymer materials. The Cole-Cole type distribution function ${ }^{29} \varepsilon^{\prime \prime}(\omega)$ described by

$$
\varepsilon^{\prime \prime}(\omega)-\varepsilon_{0}=\frac{\varepsilon_{\infty}-\varepsilon_{0}}{1+(i \omega \tau)^{a}}
$$

is related to the response function $\phi(t)$ as

$$
\varepsilon^{\prime \prime}(\omega)-\varepsilon_{0}=\left(\varepsilon_{\infty}-\varepsilon_{0}\right) \int_{0}^{\infty} \phi(t) \mathrm{e}^{-i \omega t} \mathrm{~d} t
$$

where $\varepsilon_{0}$ and $\varepsilon_{\infty}$ are complex dielectric constants at $\omega=0$ and $\omega=\infty$, and $\omega$ and $\tau$ are the frequency and the relaxation time, respectively. Solving above equation, one can obtain the function $\phi(t)$ as

$$
\begin{aligned}
\phi(t) & =\frac{a}{\tau \Gamma(1+a)}\left(\frac{t}{\tau}\right)^{-1+a}, \frac{t}{\tau} \ll 1 \\
& =\frac{a}{\tau \Gamma(1-a)}\left(\frac{t}{\tau}\right)^{-1-a}, \frac{t}{\tau} \gg 1
\end{aligned}
$$

where $\Gamma(x)$ is Gamma function. The hyperbolic function explicitly relates to distribution of the relaxation rate of the polymer main chain.

\section{Comparison by $\chi^{2}$ Values}

In general, fitting of measured data to a trial function is evaluated quantitatively by a value of $\chi^{2}$ obtained in the least square calculation. The values of $\chi^{2}$ obtained by each functions in Table II at some temperature are shown in Figure 3.

A function consisting of the product of the hyperbolic

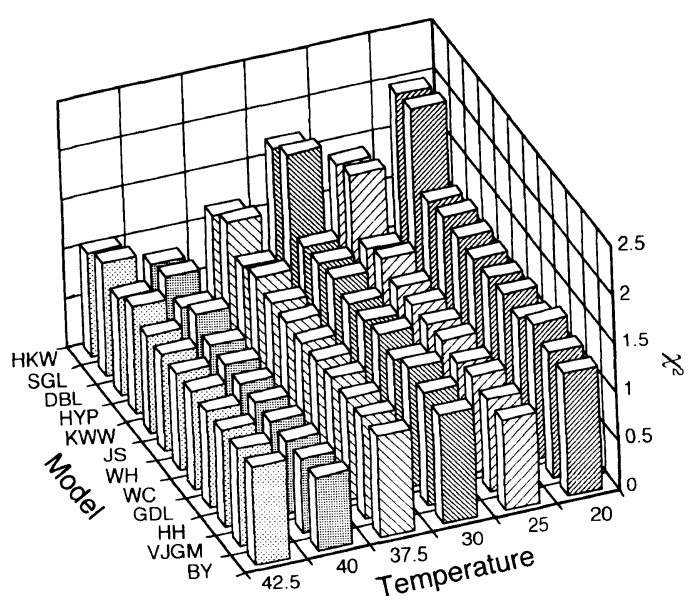

Figure 3. The values of $\chi^{2}$ calculated by each function at some temperature. HKW and SGL functions are obviously not in agreement with measured data. For other functions, it is difficult to distinguish a best function from these functions. and KWW function (HKW) proposed by Saito et al. ${ }^{26}$ and a 'Single' function are not in agreement with measured anisotropy decay curve because the values of $\chi^{2}$ are large. Thus HKW and Single functions are not appropriate for a trial function.

For functions except for HKW and Single functions, Figure 3 shows that there is little evident difference in suitability to measured data. That is to say, it is difficult to distinguish the features of a function obviously only by use of statistical value, $\chi^{2}$.

\section{Estimation of Functions by the ' $\chi^{2}$-Map Method'}

To discuss properties of reported functions to express orientational relaxation phenomena, we proposed a novel method called ' $\chi^{2}$-map method'. ${ }^{30}$ Select two parameters, and calculate the value of $\chi^{2}$ while the values of parameters are changed independently. If we consider an orthogonal coordinate system, $O-X Y Z$, with the two parameters of a function as $X$ and $Y$ coordinate axes and value of $1 / \chi^{2}$ as the $Z$ coordinate, the value of $1 / \chi^{2}$ become large when a function fits with measured data. We named such a three dimension graph as a ' $\chi^{2}$-map'. The $\chi^{2}$-map method enable us to estimate properties of a trial function from three view points; a) sensitivity of a parameter contained in the function to measured data, b) independency of parameters, and c) uniqueness of best fit values of parameters (Figure 4).

Let us assume that $p_{1}^{*}$ is determined as a best fit value of a parameter $p_{1}$. The value of $1 / \chi^{2}$ is maximum at $p_{1}=p_{1}^{*}$. The full width at half maximum (FWHM) $W\left(p_{1}\right)$ represents the sensibility of $p_{1}$. The more narrow the value of $W_{1}$ becomes, the more sensible $p_{1}$ will be. Thus a parameter which indicates the narrow profile of $1 / \chi^{2}$ is important to explain data.

If selected parameters $p_{1}$ and $p_{2}$ are independent, the
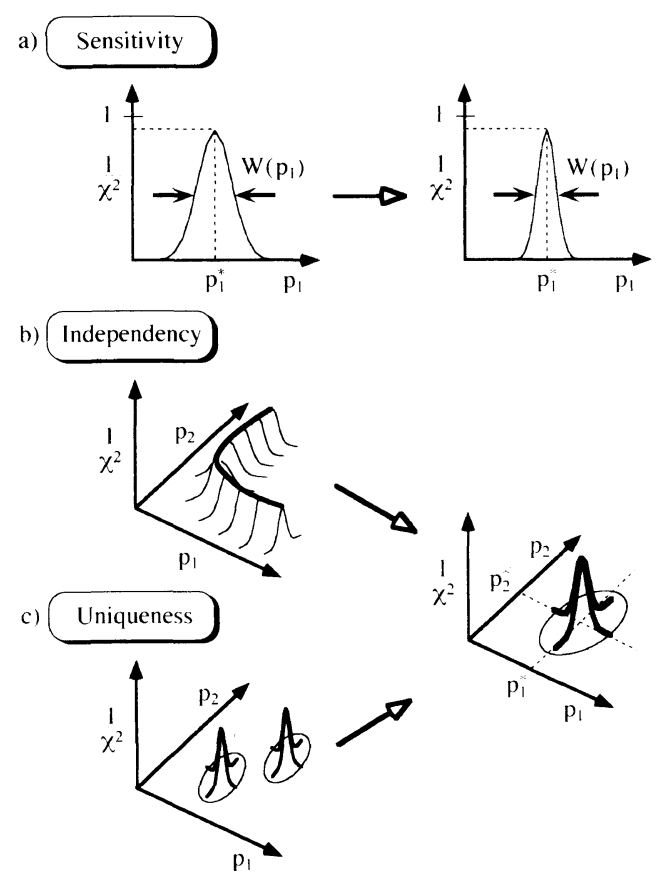

Figure 4. A $\chi^{2}$-map method. This method enable us to estimate the properties of a trial function from three view points: a) sensitivity of a parameter contained in the function to measured data; b) independency of parameters; c) uniqueness of best fit values of parameters. 


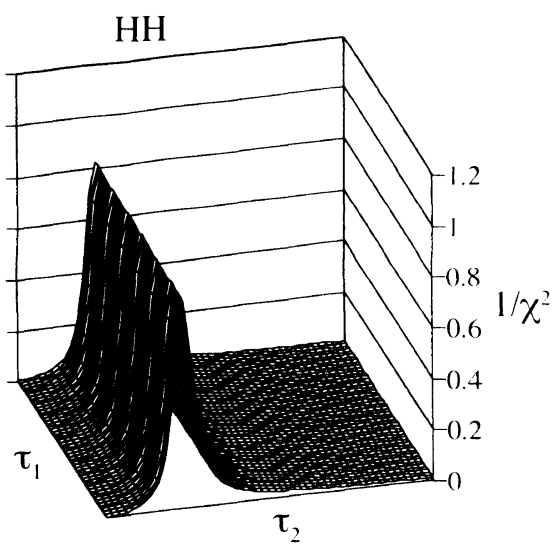

Figure 5. A $\chi^{2}$-map of $\mathrm{HH}$ function. $X, Y$, and $Z$ axes are values of $\tau_{1}, \tau_{2}$, and $1 / \chi^{2}$. A broad wall is found, so that $\tau_{1}$ is not sensitive to data.

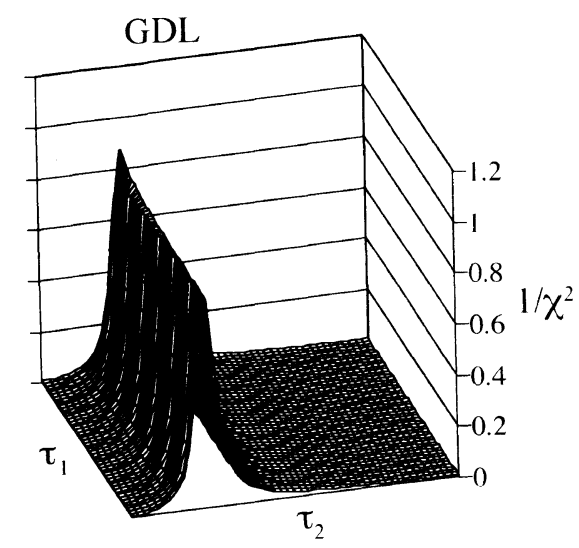

Figure 6. A $\chi^{2}$-map of GDL function. $X, Y$, and $Z$ axes are values of $\tau_{1}, \tau_{2}$, and $1 / \chi^{2}$. A broad wall is also found, so that $\tau_{1}$ is not sensitive to data.

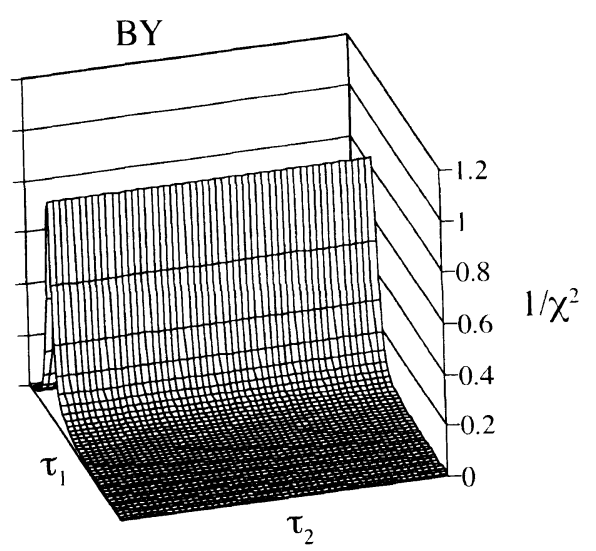

Figure 7. A $\chi^{2}$-map of BY function. $X, Y$, and $Z$ axes are values of $\tau_{1}, \tau_{2}$, and $1 / \chi^{2}$. A broad wall is also found, so that $\tau_{2}$ is not sensitive to data.

$\chi^{2}$-map will be single peak as shown in Figure 4. However if $p_{1}$ is correlated with $p_{2}$ as $f\left(p_{1}, p_{2}\right)=$ const, the shape of $\chi^{2}$-map is a horseshoe. In this case, although $p_{1}$ and $p_{2}$ are treated as independent parameters in the original theory, these parameters behave dependently when the trial function is fitted to data.

The unique results for variable parameters in a trial function give the unique peak as the $\chi^{2}$-map.

$\chi^{2}$-Maps of functions for a decay curve measured at

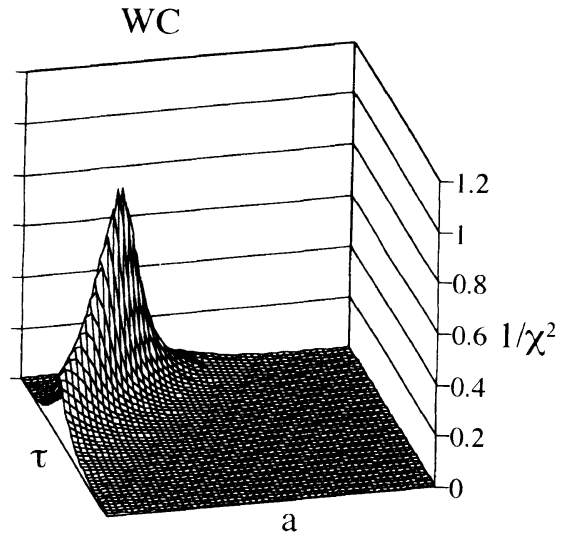

$\mathrm{W}(\tau)=6 / 50 \quad \mathrm{~W}(\mathrm{a})=6 / 50 \quad \chi^{2}=1.17$

Figure 8. A $\chi^{2}$-map of WC function. $X, Y$, and $Z$ axes are values of $\tau, a$, and $1 / \chi^{2}$. A broad and wall-like peak is found. $W\left(\tau_{1}\right)=6 / 50$; $W\left(\tau_{2}\right)=6 / 50 ; \chi^{2}=1.17$.

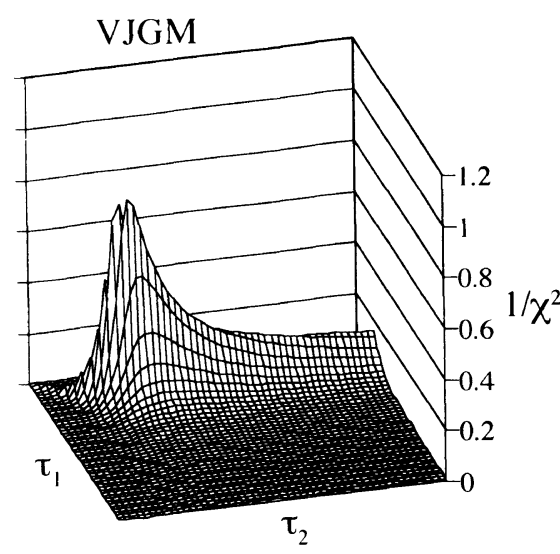

$\mathrm{W}\left(\tau_{1}\right)=5 / 50 \quad \mathrm{~W}\left(\tau_{2}\right)=10 / 50 \quad \chi^{2}=1.15$

Figure 9. A $\chi^{2}$-map of VJGM function. $X, Y$, and $Z$ axes are values of $\tau_{1}, \tau_{2}$, and $1 / \chi^{2}$. A broad peak is found. $W\left(\tau_{1}\right)=5 / 50 ; W\left(\tau_{2}\right)=10 / 50$; $\chi^{2}=1.15$.

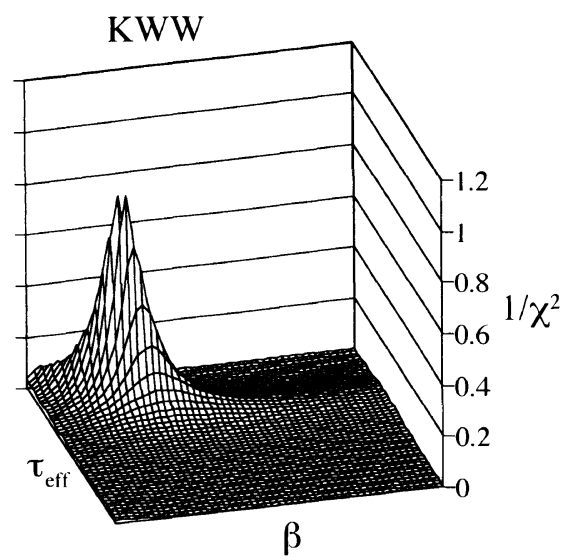

$\mathrm{W}\left(\tau_{\mathrm{eff}}\right)=5 / 50 \quad \mathrm{~W}(\beta)=5 / 50 \quad \chi^{2}=1.15$

Figure 10. A $\chi^{2}$-map of $\mathrm{KWW}$ function. $X, Y$, and $Z$ axes are values of $\tau, \beta$, and $1 / \chi^{2}$. A single peak is found, so that $\tau_{\text {eff }}$ and $\beta$ are determined independently. $W\left(\tau_{\text {eff }}\right)=5 / 50 ; W(\beta)=5 / 50 ; \chi^{2}=1.15$.

$30^{\circ} \mathrm{C}$ are shown in Figures 5-12. In these figures, parameters are varied from $0.1 \times p^{*}$ to $4 \times p^{*}$, where $p^{*}$ is the best fit of each parameter. The range of calculation was divided into fifty regions.

Figures 5, 6, and 7 indicate the $\chi^{2}$-maps of $\mathrm{HH}$, Polym. J., Vol. 28, No. 7, 1996 


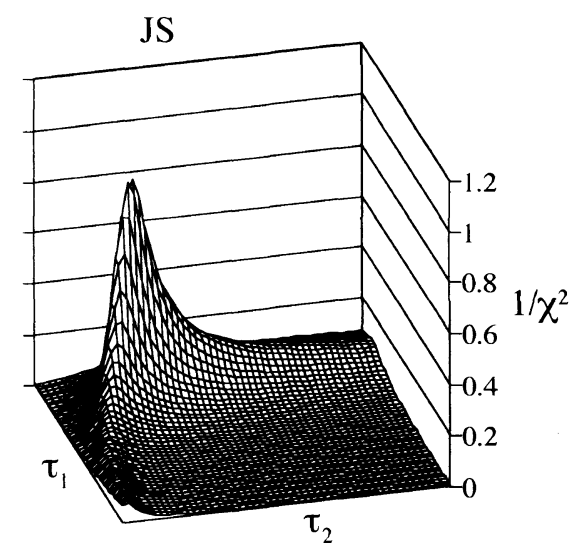

$\mathrm{W}\left(\tau_{1}\right)=8 / 50 \quad \mathrm{~W}\left(\tau_{2}\right)=9 / 50 \quad \chi^{2}=1.09$

Figure 11. A $\chi^{2}$-map of JS function. $X, Y$, and $Z$ axes are values of $\tau_{1}, \tau_{2}$, and $1 / \chi^{2}$. A single peak is found, so that $\tau_{1}$ and $\tau_{2}$ are determined independently. $W\left(\tau_{1}\right)=8 / 50 ; W\left(\tau_{2}\right)=9 / 50 ; \chi^{2}=1.09$.

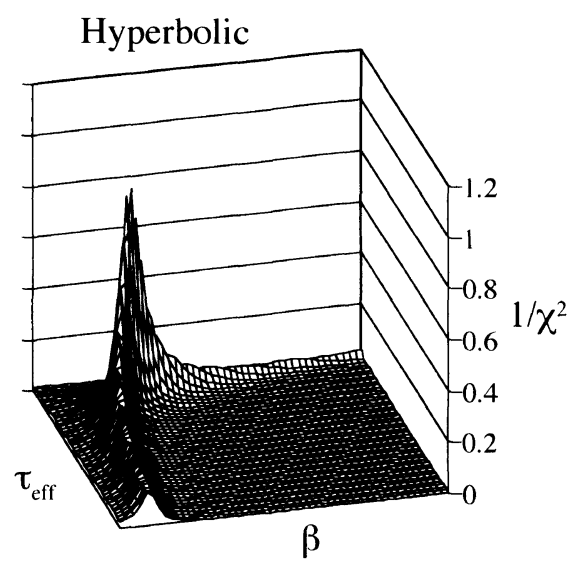

$\mathrm{W}\left(\tau_{\mathrm{eff}}\right)=3 / 50 \mathrm{~W}(\beta)=3 / 50 \quad \chi^{2}=1.11$

Figure 12. A $\chi^{2}$-map of the hyperbolic function. $X, Y$, and $Z$ axes are values of $\tau_{\text {eff }}, \beta$, and $1 / \chi^{2}$. A single and sharp peak is found, so that $\tau_{\text {eff }}$ and $\beta$ of hyperbolic function are determined independently. $W\left(\tau_{\text {eff }}\right)=3 / 50 ; W(\beta)=3 / 50 ; \chi^{2}=1.11$. Though the value of $\chi^{2}$ of the hyperbolic function is slightly larger than that of the JS fuction, parameters of the hyperbolic function are more sensitive than those of the JS function. Therefore the hyperbolic function is the most appropriate function to express fluoresccence anisotropy decay curves.

GDL, and BY functions, respectively. A broad wall is found in each map, so that $\tau_{1}$ of $\mathrm{HH}$ and GDL functions and $\tau_{2}$ of BY function are not sensitive to data. Though these functions are in agreement with measured data as is shown in Figure 3, the properties of a parameter set are not good for analysis of FADCs.

$\chi^{2}$-maps of WC, VJGM, and KWW functions have a broad and wall-like peaks as shown in Figures 8, 9, and 10. The values of FWHM reached about $5 / 50-6 / 50$, except for $W\left(\tau_{2}\right)$ of VJGM function. The value of $\chi^{2}$ of WC, VJGM, and KWW are respectively $1.17,1.15$, and 1.15. Thus KWW function is better than WC and VJGM functions.

As shown in Figures 11 and 12, $\chi^{2}$-maps of JS and hyperbolic functions have very small values of $\chi^{2}, 1.09$, and 1.11 , respectively. Thus these functions are more adequate than KWW function. Further, JS and the hyperbolic functions give a single peak in the $\chi^{2}$-map.
The best function to express FADC is JS or the hyperbolic function. The peak shown in $\chi^{2}$-map of the hyperbolic function is more sharp than that of JS function. The values of FWHM of JS function, $W\left(\tau_{1}\right)=8 / 50$ and $W\left(\tau_{2}\right)=9 / 50$, are larger than other functions. In contrast, the value of FWHM of the hyperbolic function, $W\left(\tau_{\text {eff }}\right)=3 / 50$ and $W(\beta)=3 / 50$, are the most narrow among functions. Therefore an adequate OACF to explain FADC is a hyperbolic function.

\section{CONCLUSIONS}

The hyperbolic function is the most appropriate $\mathrm{OACF}$ to express FADC of PSAPS chains because (1) hyperbolic relaxation function considers the distribution of relaxation times, (2) values of $\chi^{2}$ reach almost unity, so that measured anisotropy decay is successfully explained by hyperbolic function, (3) by use of the $\chi^{2}$-map method, it has been revealed that the parameter set of the hyperbolic function expresses sensitively FADC.

\section{REFERENCES}

1. B. Valeur and L. Monnerie, J. Polym. Sci., Polym. Phys. Ed., 14, 11 (1976)

2. A. A. Jones and W. H. Stockmayer, J. Polym. Sci., 15,847 (1977).

3. J. T. Bendler and R. Yaris, Macromolecules, 11, 650 (1978).

4. C. K. Hall and E. Helfand, J. Chem. Phys., 77, 3275 (1982).

5. J. L. Viovy, L. Monnerie, and J. C. Brochon, Macromolecules, 16, 1845 (1983).

6. T. Sasaki, M. Yamamoto, and Y. Nishijima, Macromolecules, 21 610 (1988).

7. M. D. Ediger, Ann. Rev. Phys. Chem., 42, 225 (1991).

8. S. Mashimo, Macromolecules, 9, 91 (1976)

9. W. Gronski, T. Schäfer, and R. Peter, Polym. Bull., 1, 319(1979).

10. C. Friedrich, F. Lauprêtre, C. Noël, and L. Monnerie, Macromolecules, 14, 1119 (1981).

11. D. A. Waldow, M. D. Ediger, Y. Yamaguchi, Y. Matsushita, and I. Noda, Macromolecules, 24, 3147 (1991).

12. E. V. Anufrieva and Y. Y. Gotlib, Adv. Polym. Sci., 40, 1 (1981).

13. H. Ushiki and K. Horie, "Influence of Molecular Structure on Polymer Photophysical and Photochemical Properties," Vol. 4, N. P. Cheremisinoff, Ed., Marcel Dekker, New York, 1989, p. 1.

14. B. Valeur, J. P. Jarry, F. Geny, and L. Monnerie, J. Polym. Sci., Polym. Phys. Ed., 13, 667 (1975).

15. B. Valeur, L. Monnerie, and J. P. Jarry, J. Polym. Sci., Polym. Phys. Ed., 13, 675 (1975).

16. K. Horie, K. Tomonume, and I. Mita, Polym. J., 11, 539 (1979).

17. H. Ushiki, Rep. Prog. Polym. Phys. Jpn., 35, 419 (1992).

18. P. Wahl, Biophys. Chem., 10, 91 (1979).

19. T. Nakagawa and Y. Oyanagi, "Saishou Nijyou-hou Niyoru Jikken-Data Kaiseki," Tokyo Daigaku Syuppan-kai, Tokyo, 1982.

20. T. A. Weber and E. Helfand, J. Phys. Chem., 87, 2881 (1983).

21. K. Kinoshita, S. Kawato, and A. Ikegami, Biophys. J., 20, 289 (1977).

22. R. Kohlraush, "Annalen der Physik und Chemie," Poggendorf, 1854.

23. G. Williams and D. C. Watts, Trans. Faraday Soc., 66, 80 (1971).

24. H. Takayasu, "Fractal Kagaku," Asakura, Tokyo, 1987.

25. K. Murayama, Kotai Butsuri, 21, 227 (1986).

26. R. Saito and K. Murayama, Solid State Commun., 63, 625 (1987).

27. K. L. Ngai and K. Murayama, Physica $B+C, \mathbf{1 1 7} / 118,980$ (1983).

28. G. Pfister and H. Scher, Phys. Rev. B, 15, 2062 (1977).

29. K. S. Cole and R. H. Cole, J. Chem. Phys., 9, 341 (1941).

30. H. Ushiki and F. Tsunomori, Rep. Prog. Polym. Phys. Jpn., 36, 373 (1993). 